primary beam, and a maximum in, or opposite to, that direction.

On the neutral pair hypothesis, if we only assume that the chance of ejection from an atom is equal in all directions in the plane of rotation, it may as simply be shown that the directions of minimum and maximum intensity are the same as on the previous hypothesis; but whereas on the ether pulse theory the intensity of the secondary rays in the direction of propagation of the primary is double-that in a direction at right angles, on the "neutral pair" hypothesis it varies as the cosecant of the angle which the direction of propagation of the secondary makes with that of the primary, becoming infinite along the direction of propagation of the primary. In other words, the intensity of secondary radiation varies as the density of the lines of longitude (or as the secant of the latitude) on a sphere with the secondary radiating mass at its centre, the direction of primary propagation being along the axis.

I have made experiments to test the two hypotheses, using an electroscope to compare the intensities of secondary radiation as nearly as possible in these two directions. Taking into account the finite section of the beams and consequent obliquity of the rays, the ratios on the two hypotheses would be roughly $\mathrm{I} \cdot 9: \mathrm{I}$ and $8: \mathrm{I}$, assuming perfect scattering and neglecting the effect of tertiary rays in the first case and assuming the plane of rotation to contain accurately the direction of propagation in the second. If the assumption is only approximately correct in either case, the ratio will be somewhat reduced. It is evident that great accuracy in the experiments was not essential. They, however, leave no doubt as to the conclusion, for the ratio of intensities was roughly 1.6 : Ione that might be expected on the ether pulse theory, and appears impossible on the other. It is possible that with suitable primary rays and thickness of secondary radiator, results showing more perfect scattering will be obtained.

These preliminary experiments, however, to my mind furnish quite conclusive evidence in favour of the ether pulse theory.

University of Liverpool, October 26. Charles G. Barkla.

\section{On Correlation and the Methods of Modern Statistics.}

I Do not know that much profit is likely to arise from continuing this discussion further; it appears to me to be merely unwrapping considerable convolutions in Mr. Hinks's mental attitude towards Miss Gibson and myself. The chief charge made at the British Association was that we had overlooked a curved regression line between magnitude and parallax - that now appears to have disappeared into limbo. In his first letter to Nature Mr. Hinks apparently objected to our finding " a quite significant and important" relation between parallax and proper motion, but one not more than half-way up the correlation scale. He has now discovered that "the point of most general interest" is that of colour. He charged us with stating a far-reaching suggestion on the basis of the Cape stars. It turns out now that the element in our farreaching suggestion is not the suggestion at all, but what I am prepared to assert as a fact, namely, that the magnitude of the stars " is not mainly determined by parallax or distance, but is more closely associated with colour. and thus 'probably with chemical or physical condition."; The colour and magnitude correlation is essentially that determined by Miss Gibson, $0 \cdot 3$ : the values for the spectral class and magnitude correlations run up according to the classification used to double this value and even to 0.7 . The colour and spectral class correlations reach, as we might expect, a still higher value. Meanwhile, the magnitude and parallax relation in its best determination is 0.28 . I agree with Mr. Hinks that this is a point of "general interest," and I am glad that his last letter enables me to assert it, not as "the vaguest of suggestions," which words had reference to the discontinuity of frefotency in star counts, but as a fact which may be slightly modified when more data are reduced, but is substantially correct as I have given it.

No. I983, voL. 76 ]

\section{The Interpretation of Mendelian Phenomena.}

I AM sorry Mr. Lock should mistake what I devoutly hope is a sense of proportion for a desire to belittle Mendelian work. In science clear ideas are of importance, and I wished to elicit something more definite than the vague notion that Mendelism will someday and somehow furnish a master key to the problems of heredity. I made no complaint that Mendelism " does not immediately lead to the solution of all the most difficult problems which biology affords," as Mr. Lock rather extravagantly asserts, but merely asked what conceivable bearing it can have on any problem save that of sex. By the problem of sex I mean the problem of the function of sex-or of conjugation if $\mathrm{Mr}$. Lock prefers. I confess I cannot imagine what light Mendelism has shed on the question of the alleged transmission of acquirements, and as for the " problems of the actual transmission of characters," these, as dealt with by Mendelians, are nothing other than problems of sex. That is, Mendelian experiments demonstrate nothing more than the degree in which certain characters (mutations) are transmitted or distributed under, or affected by, conditions of conjugation. 'Doubtless it is true that the majority of Mendelian cases have been observed in self-fertilised types, but I am not aware that they have ever been observed unless cross-fertilisation had previously occurred. In parthenogenesis the individual arises from an unfertilised ovum; how, then, is segregation possible? What segregates?

The evidence on which I base my assertion that there is no segregation in the mulatto is that of my own eyes. Mulattoes vary amongst themselves, but the blend is usually very obvious, and is reproduced in subsequent generations when breeding is inter se. With every infusion of European blood the negro type-skin colour, hair iexture, shape of features, and the like-grows fainter, until at length the "touch of the tar-brush" is hardly if at all perceptible; and this blending, so far as I am aware, occurs, not only in all crossed human varieties, but in other natural varieties as well. There may be exceptions; in fact, I believe there are; but blending appears to be the rule in the vast majority of instances.

How can the fact that human races have crossed more often than any other animal complicate the problem? My statement implied, not that every human race is a chaotic mixture of types, nor even that there are no pure types, but only that we have here a very large and varied mass of material on which to found our judgments. Nor did I imply that mutations are especially frequent under conditions of cultivation. I believe they are quite as common in nature. Our hospitals and asylums are full of themhare-lips, cleft-palates, club-feet, hæmophilia, colour-blindness, deaf-mutism, feeble-mindedness, and so forth. Their inheritance is usually Mendelian, but I never heard of a human mutation that was useful. I implied merely that artificial selection is founded on mutations, and that the striking difference between artificial and natural varieties indicates that natural selection is not founded on them. We know the past and present of man better than that of any other type, certainly of any natural type. Men are fond of noting wonders, and we have a written history of thousands of years; but never yet has the differentiation of a human variety by mutation been recorded. On the other hand, so surely as a human race separates into sections, between which there is little or no intercourse, gradual differentiation sets in, which, under conditions of savage warfare and very restricted intercourse, may be seen in the inhabitants of quite small tracts of country, as in New Guinea. Amongst plants and lower animals parthenogenetic types are particularly rich in varieties. "Thousands of forms may be cultivated side by side in the Botanical gardens and exhibit slight but undoubted differentiating features, and reproduce themselves truly by seed" (de Vries, "Species and Varieties," pp. 59-60). When reproduction is bi-parental, varieties are few if individuals from distant parts of a wide area are able to mate, and proportionately more numerous if intercourse is more restricted. Thus in every valley of Samoa is found a distinct variety of snails; but species of birds, mammals, and fishes which possess considerable powers of locomotion have few varieties. Is Mr. Lock able to conceive any 